\title{
Evolutionary Rule Generation Classification and Its Application to Multi-class Data
}

\author{
Susan E. Bedingfield and Kate A. Smith \\ School of Business Systems Monash University Clayton, Victoria 3168 Australia \\ \{sue.bedingfield, kate.smith\}@infotech.monash.edu.au
}

\begin{abstract}
This paper considers an evolutionary algorithm based on an information system for generating classification rules. Custom genetic operators and a multi-objective fitness function are designed for this representation. The approach has previously been illustrated using a binary class data set. In this paper we explore the possibility of using the algorithm on a multi-class data set. The accuracy of the rules produced by the evolutionary algorithm approach are compared to those obtained by a decision tree technique on the same data. The advantages of using an evolutionary classification technique over the more traditional decision tree structure are discussed.
\end{abstract}

\section{Introduction}

The classification of data into distinct groups is one of the main tasks of data mining. While many data sets involve a binary target attribute (for example good or bad credit risk), a significant number of important data sets involve the division of the data into multiple groups which refer to as multi-class data. There are many techniques for classifying both binary and multi-class data, one of the most widely used being decision trees [1]. The main reasons for the popularity of decision trees is that they are a rule based approach and they classify every data point. However for many real world data sets clear classification boundaries may not exist and this may be useful information that becomes lost when adopting a technique that forces such clear cut classifications. In addition decision trees only explore a subset of all possible rules due to the greedy nature of their decision making [2]. The generation of branches in a decision tree is dependent upon previous splitting, each split seeks to maximise the accuracy of the classification at that level rather than looking ahead to consider the entire search space. Given this limitation, and the attractiveness of a rule based approach to classification for ease of interpretability, we consider the benefits of using an evolutionary approach to rule generation.

In this paper we first introduce an evolutionary classification technique and test its viability on a database with a multi-class target attribute. We then compare the evolutionary classification technique with See5, a widely used decision tree software package used for classification. The evolutionary technique used in this paper has been reported upon in [3],[4] and [5]. So far the technique has been used a database 
with a binary consequence attribute - the well known German credit scoring data. However the technique can be generalised to any number of classes corresponding to the number of attribute values belonging to the consequence attribute. In the example that follows we use the well known Iris database with 3 classes.

We then compare the evolutionary technique implemented in CS-classifier with the decision tree algorithm implemented by See5, both in terms of the classification rules generated and the error rates observed by testing the rules on randomly generated test sets.

CS-classifier is a global evolutionary search technique that makes no assumptions about the attribute values, the consistency or accuracy of the data. See5, on the other hand, is a greedy search algorithm which forces classification irrespective of the data quality.

Our aim is to compare the performance of the two methods and determine if the more flexible nature of the evolutionary approach leads to more insightful and accurate rules.

\section{The Evolutionary Algorithm}

\subsection{Rules and Rule Structure}

Each population consists of a collection of rule sets and each rule set is simply a collection of rules, the number of rules in each rule set is specified as one of the parameters in the evolutionary algorithm. Each individual rule is made up of a set of atoms. An atom is a structure of the form $(\alpha, \beta, \gamma)$ where $\alpha$ represents an one of the attributes, $\beta$ is a comparison operator $(\leq, \geq,=)$ and $\gamma$ a is number within the range of values assumed by the attribute $\alpha$.

A rule has a structure of the form (atom1, atom $2, \ldots$, atom $N$ ) where $N \leq M$ and $M \geq 2$ is a parameter defining the upper bound on the number of atoms allowed to make a rule. A restriction is placed on the number of atoms allowed to ensure that the rules are easily interpreted and understood by humans. For each rule, atom 1, atom $_{2}, \ldots$, atom $_{N-1}$ have a condition attribute as the attribute component, and atom $_{N}$ has a decision attribute as its attribute component.

Note that a particular attribute may appear more than once in an atom, for example when an upper and lower bound for a specific attribute is required. For example, a rule might be:

(petal length $>50$, petal width $<=60$, species $=$ iris setosa) .

A rule system, $\mathfrak{R}$, comprises a finite set of rules $\left\{\right.$ rule $_{1}$, rule $_{2}, \ldots$, rule $\left._{k}\right\}$ where $1 \leq k \leq K$, $K$ being a parameter defining the number of allowable rules in a rule set. The evolutionary algorithm population consists of $P$ rule systems each containing $\mathrm{k}$ individual rules. 


\subsection{Genetic Operators}

The genetic operators used in this paper were an elitist selection criteria, crossover and mutation.

\section{Crossover}

Crossover between individual rule systems is accomplished randomly based on a fixed crossover probability $\mathrm{P}_{\mathrm{c}}$. The first $C$ rules from each rule system are selected and swapped.(The starting point for the swap is also determined as part of the crossover process). $C$ may vary from 0 to $M$ where $M$ is half the size of the smaller rule system. The rule systems produced by the crossover are added to the population, thus temporarily increasing its size.

\section{Mutation}

Mutation occurs to the rules within the individual rule bases and takes various forms random changes to the components of each atom, swapping one or more atoms between two rules, deleting a rule, adding a new rule. The probabilities of each type of mutation is parameterised .

\section{Selection}

The selection criteria used is elitist - the best $P$ rule sets of the current generation are selected to form the next generation.

\subsection{Fitness Function Components}

There are several measures of fitness of the individual rules in each rule base that we are interested in recording and improving over time. We first consider accuracy measures - both row and column accuracy - of the resulting confusion matrix. The particular information system we are experimenting with has a decision attribute with 3 possible values. This leads to 3 row accuracy measures $M_{1}, \ldots . M_{3}$, and 3 column accuracy measures $M_{4}, \ldots . . M_{6}, M_{1}, \ldots . . M_{3}$, effectively measure the coverage by the rule system of the 3 classification groups. Whereas $M_{4}, \ldots \ldots, M_{6}$ measure the accuracy of the predictions for each classification group. These 6 expressions should optimally be equal to 1 . Analagous to the three accuracy measures $M_{1}, \ldots \ldots M_{3}$, are three measures of inaccuracy, $M_{7}, \ldots . . M_{9}$, designed to reduce incorrect classifications.

We let $O$ represent the set of data points in the Iris database, $A$ the set of attributes. Let $V_{d}=\left\{v_{1}, v_{2}, v_{3}\right\}$ represent the set of attribute values that the target attribute can take. If $v \in \mathrm{V}_{d}$, where $d$ is the target attribute, we define $O_{v}$ to be the subset of $O$ whose decision attribute value is $v$.

If we let $\Re$ represent a rule set and if $\rho \in \Re$, then we define $O_{\rho}$ to be the subset of $O$ whose members satisfy the condition attribute values associated with $\rho$ and $O_{\rho, \mathrm{d}(\rho)}$ to be the subset of $O$ whose members satisfy the rule $\rho$ (i.e. they satisfy the condition and target values for $\rho$ ).

We can express the measures $M_{1}, \ldots . . M_{9}$, as follows: 


$$
M_{i}, i=1, \ldots, 3 \text { are defined as } \frac{\operatorname{Card}\left(O_{v_{i}} \cap \bigcup_{d(\rho)=v_{i}} O_{\rho}\right)}{\operatorname{Card}\left(O_{v_{i}}\right)}
$$

\section{Combining Fitness Function Components}

The total fitness function for the evolutionary algorithm can be represented as the weighted function of the nine components $M_{1}-M_{9}$ defined above, and attempts to generate rules over time with maximal coverage, maximal accuracy, minimal contradictions.

\section{The Fitness Function}

The fitness function used for the work presented in this paper has the following form:

$$
\begin{gathered}
F=\lambda F^{1}+(1-\lambda) F^{2} \text { where } 0<\lambda<1 \text { and } \\
F^{1}=\left(\frac{\sum_{i=1}^{6} M_{i}}{1+\sum_{i=7}^{9} M_{i}}\right), \quad F^{2}=\left(\sum_{i=1}^{6} M_{i}+\sum_{i=7}^{9} \frac{1}{1+M_{i}}\right)
\end{gathered}
$$

This form of the fitness function has been discussed previously in [5]. It has been observed that the component $F^{l}$ if used solely as the fitness function tends to induce rules which are highly accurate at the cost of overall coverage. Whereas if the component $F^{2}$ is used as the fitness function, the rule systems generated tend to have a 
higher classification coverage at the cost of classification accuracy. A linear combination of the two components $F^{l}$ and $F^{2}$ using the parameter lambda has been used to introduce greater versatility to the fitness function.

\section{Parameter Values}

For the experiments reported upon in this paper, the fixed parameter values listed in table 1 were used

Table 1. Parameter values

\begin{tabular}{|l|l|l|}
\hline Parameter name & Value & Explanation \\
\hline PopSize & 10 & The number of rule sets in each population \\
\hline MaxAtoms & 6 & $\begin{array}{l}\text { The maximum initial number of atoms in each } \\
\text { rule }\end{array}$ \\
\hline RulesPerSet & 10 & The initial number of rules in each rule set \\
\hline ChgComponent & 0.5 & Probabilty of changing a component of an atom \\
\hline SwapAtom & 0.2 & Probabilty of swapping atoms between rules \\
\hline DelRule & 0.5 & Probabilty of deleting rule from a rule set \\
\hline & & $\begin{array}{l}\text { The probability of adding a new rule to a rule set } \\
\text { - in this case a new rule is always added. Any } \\
\text { empty rules are deleted. }\end{array}$ \\
\hline AddRule & 1 & Probability of adding an atom to a rule \\
\hline AddAtom & 0.2 & Probabilty of crossing two rulesets \\
\hline
\end{tabular}

\section{Experimental Design and Data Set}

\subsection{The Data}

The data on which the rule system generation is based is the Iris database which originated with Fisher [6]. This data set was chosen because the target attribute is multiclass and also the data set has been widely reported upon in the literature. The data contains 150 examples of irises belonging to three different species, iris setosa, iris versicolor and iris virginica. There are 5 attributes available for modelling the iris species. These include species, sepal width and length, petal width and length. There is an equal distribution of cases amongst the three species. For this experiment a 5 fold cross validation was performed on the data to establish the viability of the technique on a dataset with a non-binary valued consequence attribute. A 5 fold cross validation was then performed using See 5 with the same test and training sets, and a comparison was made between the two techniques.

\section{5-Fold Cross-Validation}

Five distinct training and test sets containing $80 \%$ and $20 \%$ of the total data set respectively were randomly generated from the data set. Each pair of sets in turn was used to test the classification accuracy of the of the rule system generated by CS- 
Classifier and compared with the classification accuracy of decision tree generated by See5.

CS-classifier was run for each training set, for each value of $\lambda=0, .2, .4 .6 .8,1$. For the following comparisons, the most accurate rule set for each corresponding test set was then selected.

There is one major difference in the rule sets that are generated by CS-classifier and See5.

The rules generated by See5 provide exactly one classification for all data points in the training/test sets. Whereas the rules generated by CS-classifier may provide zero, one or many classifications for any given data point in the training/test sets. Thus there are

there are four ways of calculating the error rates for the CS-classifier predictions:

1. error rate $=$ (number of misclassifications $) /($ total number of data points classified)

2. error rate $=$ (number of misclassifications $) /($ total number of data points). In this case, if for a specific data point there is no rule providing a prediction, this is interpreted as a misclassification.

3. error rate $=$ (total number of misclassifications using most accurate rule)/(total number of classifications). In this case, if for a specific data point there is a rule which provides a correct classification and there are no rules of greater accuracy providing an incorrect classification, then this is interpreted as a correct classification.

4. error rate $=$ (total number of misclassifications)/(total number of data points). In this case also, if for a specific data point there is a rule which provides a correct classification and there are no rules of greater accuracy providing an incorrect classification, then this is interpreted as a correct classification.

\section{Results}

The error rates for training and test sets using each of the four interpretations are listed in tables 2 and 3 respectively.

Table 2. Error rates for training sets

\begin{tabular}{|l|r|l|l|l|l|l|c|}
\hline Method & Set 1 & Set 2 & Set 3 & Set 4 & Set 5 & mean & $\begin{array}{c}\text { Standard } \\
\text { deviation }\end{array}$ \\
\hline See5 & 2.5 & 1.67 & 2.5 & 2.5 & 2.5 & 2.33 & 0.37 \\
\hline $1 . \quad$ CS-classifier & 3.57 & 1.75 & 4.00 & 5.98 & 5.08 & 4.08 & 1.61 \\
\hline 2. $\quad$ CS-classifier & 10.00 & 6.67 & 20 & 8.33 & 6.67 & 10.33 & 5.58 \\
\hline 3. $\quad$ CS-classifier & 2.68 & 0 & 3 & 4.27 & 3.39 & 2.67 & 1.61 \\
\hline $4 . \quad$ CS-classifier & 9.17 & 5 & 19.17 & 6.67 & 5.00 & 9.00 & 5.93 \\
\hline
\end{tabular}


Table 3. Error rates for test sets

\begin{tabular}{|l|l|l|l|l|l|l|c|}
\hline Method & Set 1 & Set 2 & Set 3 & Set 4 & Set 5 & mean & $\begin{array}{c}\text { Standard } \\
\text { deviation }\end{array}$ \\
\hline See5 & 0 & 13.33 & 6.67 & 6.67 & 13.33 & 8.00 & 5.00 \\
\hline $1 . \quad$ CS-classifier & 0 & 0 & 6.67 & 3.33 & 6.9 & 3.38 & 3.04 \\
\hline 2. $\quad$ CS-classifier & 0 & 10 & 6.67 & 3.33 & 10 & 6.00 & 3.89 \\
\hline $3 . \quad$ CS-classifier & 0 & 0 & 3.33 & 0 & 3.45 & 1.36 & 1.66 \\
\hline $4 . \quad$ CS-classifier & 0 & 10 & 3.33 & 0 & 6.67 & 4.00 & 3.89 \\
\hline
\end{tabular}

Observing Tables 2 and 3 we see that Training accuracy results for See5 are much more consistent than those for CS-classifier. Also, error rates for See5 on the training sets appear lower than on the test sets. Conversely, error rates for CS-classifier on the test sets appear lower than on the training sets.

An analysis of variance was performed between the error rates for See 5 on the test sets and the error results for CS-classifier using the four separate methods for assessing the error rate. It was found that for methods 1,2 and 4 there is no statistical difference in error rates, (p-values being .081, .456 and .152 ) between the two techniques. However if method 3 is used to assess the error rate for CS-classifier, the analysis indicates an improvement in the classification accuracy (with p-value .011).

For training set 1 , the rule derived from the decision tree generated by See5 is as follows:

If petal-length $<=190$ : Iris-setosa $(40,0)$

Else if petal-length > 190:

if petal-width $>170$ : Iris-virginica $(39,1)$

else if petal-width $<=170$ :

if .petal-length $<=490$ : Iris-versicolor $(35,1)$

else if petal-length $>490$ :

if .petal-width $<=150$ : Iris-virginica $(3,0)$

else if petal-width $>150$ : Iris-versicolor $(3,1)$

The numbers in brackets indicate the number correctly classified followed by the number misclassified by the rule at each branch. This rule completely classifies test set 1 correctly.

In comparison, one of the rule sets generated by CS-classifier using training set 1 is as follows:

Rule 1: (petal_length<239) and (petal_width<=95) then Iris setosa $(40,0)$

Rule 2: (petal_width>91) and (petal_width $<=172$ ) and (sepal_length $<=704$ ) then Irisversicolor $(40,4)$

Rule 3: (petal_width>182) then Iris-virginica $(29,0)$

Rule 4: (petal_length>517) then Iris-virginica $(28,0)$

This rule set also correctly classifies test set 1 . The numbers in brackets indicate the total number classified followed by the number misclassified by the rule. Some brief observations on each of the rules follows. 


\section{Rule 1 of CS-Classifier}

In this case either condition for rule 1 of CS-classifier would be sufficient to classify Iris setosa correctly with complete accuracy on the training set. The minimal class boundary for petal length of 190 has been found by See5. however the class boundary found by CS-classifier is 239 rather than 190. This is because for the purposes of classification, 239 works just as well so that fitness measure is not improved with a value of 190 rather than 239 (in fact any value between 190 and 299 would work just as well).

\section{Rule 3 and Rule 4 of CS-Classifier}

Rules 3 and 4 classify Iris virginica completely with complete accuracy on both the training set and test set. There is some classification overlap here - i.e. both Rule 3 and Rule 4 correctly classify some of the same data points in the training and test sets.

\section{Rule 2 of CS-Classifier}

In this case all three conditions in rule 2 are necessary to ensure the same level of accuracy $(90 \%$ on the training set and $100 \%$ on the test set).

\section{Conclusion}

We have demonstrated the viability of the evolutionary algorithm on a multi-class data set. We have also demonstrated that the classification rules generated by CSclassifier compare favourably, in terms of accuracy, with those generated by See5. See5 uses a greedy search algorithm which forces classification rules on the training data. This can be regarded as an advantage as all data points in the test set can then be given a classification. However, the disadvantages of this is that the rules may well provide less accurate classifications on test set and the rules produced may provide less insight into the true nature of the data divisions. Conversely, although CSclassifier is encouraged to classify by the components $M_{1}, . ., M_{3}$ in the fitness function, it is not forced to classify and will not do so if it means reducing the value of the fitness function. CS-classifier has the ability to recognise the possible uncertain and contradictory nature of a data set. The result of this is that using the rules generated by CS-classifier, we see a decrease in the classification errors on the test set compared to the training set, rather than the increase of classification errors generated on the test set compared with the training that we see when we use the rule generated by See5. The ability of CS-classifier to provide accurate classification rules can be enhanced by weighting the fitness function components $M_{l}-M_{9}$. These weights can be defined in a way that reflects the actual misclassification costs associated with each of the components [5]. The technique also lends itself to hybridisation with fuzzy and rough set approaches, which will be the subject of future work. 


\section{References}

1. Quinlan, J. Ross, "C4.5:Programs for Machine Learning”, The Morgan Kaufmann Series in Machine Learning, Pat Langley, Series Editor, 1992.

2. Information Discovery Inc., "Rules are much More than Decision Trees", http://www.datamining.com/trees.htm, access date: 10 Dec., 2002.

3. Bedingfield, S. E. and Smith, K. A., "Evolutionary Rule Generation in an Information System", in Dagli, C. H. et al. (eds.), Intelligent Engineering Systems Through Artificial Neural Networks, ASME Press, NY, volume 9, pp. 485-492, 1999.

4. Bedingfield, S. and Smith, K. A., "A Comparison of Fitness Functions for Evolutionary Rule Generation", in M. Mohammadian (ed.), Advances in Intelligent Systems: Theory and Applications, IOS Press, 2000.

5. Bedingfield, S. and Smith, K. A., "Evolutionary rule generation and its application to credit scoring", L.Reznik and V.Kreinovich (eds.), Soft Computing in Measurement and Information Acquisition, Physica-Verlag, Heidelberg, accepted for publication, 2001.

6. Fisher,R.A. "The use of multiple measurements in taxonomic problems" Annals of Eugenics, 7, Part II, 179-188 (1936). 